\title{
Electrophysiologist shows a cation channel function of MLKL
}

Cell Research (2016) 26:643-644. doi:10.1038/cr.2016.64; published online 27 May 2016

\begin{abstract}
Whether mixed lineage kinase domain-like (MLKL) mediates necroptosis by forming ion channel, noneselective pore, or simply by disrupting plasma membrane is unclear. In a paper published in recent issue of Cell Research, Xia et al. showed that MLKL functions as a novel class of cation channel.

Necroptosis is a type of programmed
\end{abstract} cell death with necrotic morphology. In recent years, scientists have provided us with many details in molecular mechanism of necroptosis and uncovered its function in many physiological and pathological processes. Necroptosis is controlled by a signaling complex called necrosome (also known as complex IIb). The core components of this complex are receptor interacting protein 1 (RIP1) and RIP3, though RIP1 is not always indispensable. RIP3 homo-interaction leads to RIP3 auto-phosphorylation in necrosome. Auto-phosphorylated RIP3 recruits MLKL and phosphorylates MLKL[1]. MLKL is a pseudokinase with an N-terminal domain, which is responsible for triggering necroptosis, and a C-terminal kinase-like domain, which is inhibitory to MLKL function. Further study revealed that after being phosphorylated by RIP3 at the activation loop of the kinase-like domain, MLKL undergoes oligomerization and translocation onto the plasma membrane to execute cell death [2-5]. The affinity for phosphatidylinositol phosphates is believed to play a role in the interaction of MLKL with plasma membrane $[4,5]$. There are several models on the mechanism of how MLKL functions on plasma membrane: MLKL forms permeable pores on plasma and intracellular membranes, allowing for membrane disruption and subsequent necroptosis; MLKL targets its downstream TRPM7 to mediate calcium influx that causes necroptosis; MLKL functions on plasma membrane to increase sodium influx, which causes osmotic pressure and then disrupts plasma membrane. These models, however, still need to be further experimentally verified.

In a recent issue of Cell Research, Xia et al. demonstrated that MLKL can function as cation channel in vitro [6]. Initially, by using a planar lipid bilayer recording technique, the authors found that MLKL phosphomimetic mutant $\left(\mathrm{MLKL}^{\mathrm{E} / \mathrm{D}}\right.$ ) forms ion channels. They further defined that MLKL channels are permeable to monovalent ions $\mathrm{Na}^{+}$ and $\mathrm{K}^{+}$rather than $\mathrm{Cl}^{-}$. To take one step further, they evaluated the permeability of MLKL channels to divalent cations and demonstrated that MLKL channels are preferentially permeable to $\mathrm{Mg}^{2+}$ than to $\mathrm{Ca}^{2+}$ in the presence of $\mathrm{Na}^{+}$and $\mathrm{K}^{+}$. Taking advantage of the whole-cell patch clamp technique, the authors resolved positive correlations among the whole-cell current density, depolarization of the membrane potential and necroptosis, and suggested that MLKL channel activity is a critical determinant for executing necroptosis.

By comparison of different MLKL truncations, the authors illustrated that the N-terminal domain of MLKL, similar to $\mathrm{MLKL}^{\mathrm{E} / \mathrm{D}}$, suffices to function as channel in a lipid bilayer, among which $\alpha$-helices $\mathrm{H} 1$ to $\mathrm{H} 5$ are responsible for the conductivity, while helix $\mathrm{H} 6$ and the following segments are required for its channel function. Moreover, by using substituted cysteine accessibility mutagenesis (SCAM) strategy and the planar lipid bilayer system, the authors were able to determine the configuration of the six helices in membrane, that is, helices $\mathrm{H} 1, \mathrm{H} 2, \mathrm{H} 3, \mathrm{H} 5$ and $\mathrm{H} 6$ are transmembrane, while helix $\mathrm{H} 4$ locates in the cytoplasm. This finding of MLKL being a five-transmembrane spanning domain protein, together with its preferential permeability, distinguishes it from other ion channels, such as TRPM6, TRPM7 and voltage-gated potassium (Kv) channels. The proposed five-transmembrane segment topology of MLKL differs from most people's prediction that the opening of the Nterminal bundle in MLKL is required for it to function in necroptosis and $\mathrm{H} 6$ acts as a plug to prevent opening of the bundle [7-10].

Taken together, the study by Xia et $a l$. provides a new working model for the function of MLKL in necroptosis. However, it is still unclear how a $\mathrm{Mg}^{2+}$ channel functions to trigger necroptosis and whether the ion selectivity of MLKL is required for necroptosis. Moreover, further investigation is needed to confirm the five-transmembrane structure of MLKL in plasma membrane of cells. Besides, the authors have not addressed an even more important issue, that is, the structure of MLKL oligomer based on their five-transmembrane domain model.

Yingying Zhang ${ }^{1}$, Jiahuai $\operatorname{Han}^{1}$ 
${ }^{1}$ State Key Laboratory of Cellular Stress Biology, Innovation Center for Cell Signaling Network, School of Life Sciences, Xiamen University, Xiamen, Fujian 361005, China

Correspondence: Jiahuai Han

E-mail: jhan@xmu.edu.cn

\section{References}

1 Sun L, Wang H, Wang Z, et al. Cell 2012;
148:213-227.

2 Cai Z, Jitkaew S, Zhao J, et al. Nat Cell Biol 2014; 16:55-65.

3 Chen X, Li W, Ren J, et al. Cell Res 2014; 24:105-121.

4 Dondelinger Y, Declercq W, Montessuit S, et al. Cell Rep 2014; 7:971-981.

5 Wang H, Sun L, Su L, et al. Mol Cell 2014; 54:133-146.

6 Xia B, Fang S, Chen X, et al. Cell Res 2016;
26:517-528.

7 Murphy JM, Czabotar PE, Hildebrand JM, et al. Immunity 2013; 39:443-453.

8 Hildebrand JM, Tanzer MC, Lucet IS, et al. Proc Natl Acad Sci USA 2014; 111:1507215077.

9 Su L, Quade B, Wang H, et al. Structure 2014; 22:1489-1500.

10 Quarato G, Guy CS, Grace CR, et al. Mol Cell 2016; 61:589-601. 